\title{
Mining Top-K Largest Tiles in a Data Stream
}

\author{
Hoang Thanh Lam ${ }^{1,3}$, Wenjie Pei ${ }^{1}$, Adriana Prado ${ }^{2}$, Baptiste Jeudy ${ }^{2}$, \\ and Élisa Fromont ${ }^{2}$ \\ 1 Technische Universiteit Eindhoven, 5612 AZ, Eindhoven, The Netherlands \\ 2 H. Curien Lab, UMR CNRS 5516, Université de St-Etienne, Université de Lyon, \\ France \\ 3 IBM Research Lab, Damastown, Dublin, Ireland
}

\begin{abstract}
Large tiles in a database are itemsets with the largest area which is defined as the itemset frequency in the database multiplied by its size. Mining these large tiles is an important pattern mining problem since tiles with a large area describe a large part of the database. In this paper, we introduce the problem of mining top- $k$ largest tiles in a data stream under the sliding window model. We propose a candidatebased approach which summarizes the data stream and produces the top- $k$ largest tiles efficiently for moderate window size. We also propose an approximation algorithm with theoretical bounds on the error rate to cope with large size windows. In the experiments with two real-life datasets, the approximation algorithm is up to hundred times faster than the candidate-based solution and the baseline algorithms based on the state-of-the-art solutions. We also investigate an application of large tile mining in computer vision and in emerging search topics monitoring.
\end{abstract}

\section{Introduction}

Mining frequent patterns is an important research topic in data mining. However, instead of focusing on exhaustive search to find all possible frequent patterns, many works are now focusing on designing methods that are not only efficient in the context of very big data but also limit, e.g. with constraints or with new interestingness measures, the number of patterns output by these algorithms.

Area is a measure of pattern interestingness defined as a pattern's frequency in the database multiplied by its size. It has been shown that in some applications such as in role mining [10,16] where the idea is, given a set of users and a set of permissions, to find a minimum set of roles such that all users will be assigned a role for which some permissions will be granted, mining roles is equivalent to a variant of mining itemsets with large area in a database.

Recent applications produce a large variety of transactional data streams, such as text stream from twitter 1 or video stream in which video frames can be converted into transactions [6]. In the context of data streams, data usually arrive continuously with high speed, hence requiring efficient mining techniques for summarizing the data stream and keeping track of important patterns.

1 WWW.twitter.com

T. Calders et al. (Eds.): ECML PKDD 2014, Part II, LNCS 8725, pp. 82 972014.

(C) Springer-Verlag Berlin Heidelberg 2014 
In this paper we tackle the problem of mining the top- $k$ largest tiles, i.e. the $\mathrm{k}$ closed itemsets with the largest area in a stream of itemsets. The problem of mining the largest tile in a database is well-known to be NP-hard and inapproximable [7. Therefore, the straightforward approach that recalculates the set of top- $k$ largest tiles from scratch every time a sliding window is updated is not efficient. To deal with this situation, we first introduce in Section 4 a candidate-based approach which incrementally summarizes the stream by keeping the itemsets that can be the top- $k$ largest tiles in some future windows. For each candidate, an upper-bound and a lower-bound of the area in any future window are kept. These bounds are used to prune the candidates when they cannot be the top- $k$ largest tiles in any future window. In doing so, the candidate-based algorithm is more efficient than the straightforward approach because updating is cheaper than recalculating the top- $k$ tiles from scratch.

However, when the widow size is large, the candidate-based algorithm is inefficient because the summary grows very quickly. Therefore, we introduce an approximation algorithm with theoretical bounds on the error rate. In the experiments with two real-life datasets presented in Section 6, the approximation algorithm is two to three orders of magnitude faster and more accurate than the candidate-based solution and the baseline algorithms created based on the stateof-the-art solutions for the top- $k$ largest tiles mining. We also discuss potential applications of mining large tiles for object tracking in video and emerging search topics monitoring problems.

\section{Related Works}

Recent works [12917] propose approaches to solve the redundancy and trivial patterns issues in frequent pattern mining. For instance, the first two works focuse on finding a relevant or concise representation of sets of frequent patterns. Meanwhile, the last work solves the aforementioned issues by proposing a MDLbased approach that finds patterns compressing the database well. In all these cases as well as in ours, the purpose is to limit the output of the algorithms to a set of useful patterns.

The problem of mining large tiles has already been tackled in 10,1613. The authors of the two first papers show that the problem of finding roles is equivalent to variants of the tiling database problem. The last paper shows how tiles can be used to understand complex proteins by identifying their subunits. The authors of 4. also showed that the dense rectangles in a binary matrix seem to correspond to interesting concepts. Therefore, mining large tiles may help to identify those interesting concepts from the databases. In [15] the authors investigate how to output the set of tiles in a tree representation which is easily interpretable by the users. Tiles are also used to identify and characterize anomalies in a database in [14.

In many applications, data arrives in a streaming fashion with high speed [1. Besides the popularity of data streams in many applications, the temporal aspect of the patterns such as the evolution of patterns overtime [1] provides 

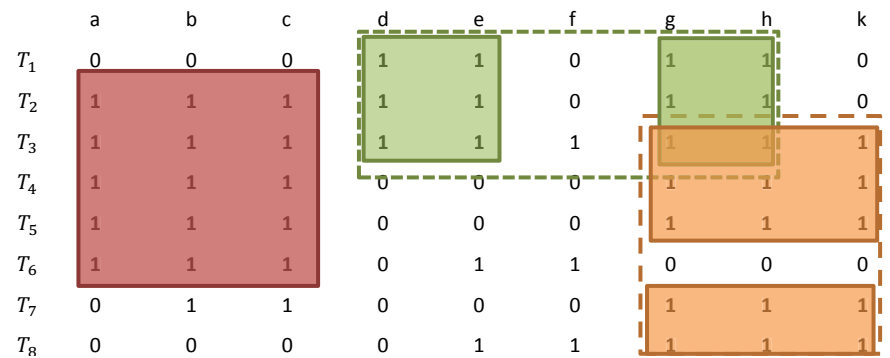

Fig. 1. An example of large tiles in a window with $w=8$ transactions

useful insights about the data for the users. Despite the importance of data stream paradigm, there is no work yet addressing the problem of mining large tiles in a data stream. The algorithms introduced in this paper are, to the best of our knowledge, the first to solve the problem of mining the top- $k$ largest tiles in a stream of itemsets under the sliding windows model.

\section{Problem Definition}

Let $\Sigma$ be an alphabet of items, a transaction $T$ is an itemset $T \subseteq \Sigma$. Let $S=$ $T_{1} T_{2} \cdots T_{n}$ be a stream of transactions where each transaction $T_{t}$ is associated with a timestamp $t$ indicating the order of transaction generation.

In this work we consider the sliding window model in which a window of the $w$ most recent transactions is monitored. A sliding window of size $w$ at time point $t$ denoted by $W_{t}$ is a sequence of $w$ consecutive transactions in the data stream, i.e. $W_{t}=T_{t-w+1} \cdots T_{t-1} T_{t}$.

For any given itemset $I \subseteq \Sigma$ the frequency of $I$ in a sliding window $W_{t}$, denoted by $f_{t}(I)$, is defined as the number of transactions in $W_{t}$ that are the supersets of $I$. Let $|I|$ be the cardinality of the set $I$, the area of $I$ in the window $W_{t}$ denoted by $A_{t}(I)$ is defined as $A_{t}(I)=f_{t}(I) *|I|$.

An itemset $I$ is closed in a window $W_{t}$ if there is no superset of $I$ with the same frequency as $I$ in the window $W_{t}$. Such itemsets are usually called large tiles in the literature [7. Itemsets that are not closed correspond to sub-tiles of large tiles and are thus not interesting for our problem. From now on we use the term tiles to refer to closed itemsets.

Example 1 (Large tiles). Figure 1 shows a window with 8 transactions represented as rows of a binary matrix. In each row, an element is equal to 1 if the corresponding item belongs to the transaction. In this figure, three large tiles $a b c$, degh and $g h k$ with respective area 15, 12 and 15 are highlighted. Large tiles are the maximal sub-matrices containing only 1.

The problem of stream tiling can be formulated as :

Definition 1 (Stream Tiling). Given a data stream of itemset transactions $S$, a parameter $k$ and a window size $w$, the stream tiling problem consists in computing the $k$ tiles with the largest area in every window of size $w$. 


\section{Algorithms}

It was proven that the problem of mining the largest tile in a database is NPComplete and is even inapproximable [7. Therefore, recalculation of the top$k$ largest tiles from scratch every time the window is updated is very timedemanding. In this section we discuss efficient solutions for the given problem under the streaming context.

\subsection{Candidate-Based Algorithm}

We first discuss an exact algorithm named cTile which maintains a summary of the sliding window containing candidate itemsets that potentially can become top- $k$ largest tiles in any future window. This summary is designed such that it can avoid expensive recalculation of the set of largest tiles from scratch when the window is sliding.

The general idea of the algorithm is as follows: at every transaction $T_{i}$ we keep a candidate list $C_{i}$ of all closed subsets of the given transaction which can become a top- $k$ tile in a future sliding window. In order to identify these closed itemsets, we keep a lower-bound and an upper-bound on the area of these itemsets in any future sliding window which contains $T_{i}$. These bounds will be used to infer which itemsets can be top- $k$ tiles and which sets for sure cannot be top- $k$ tiles in any future window (thus can be removed from the summary).

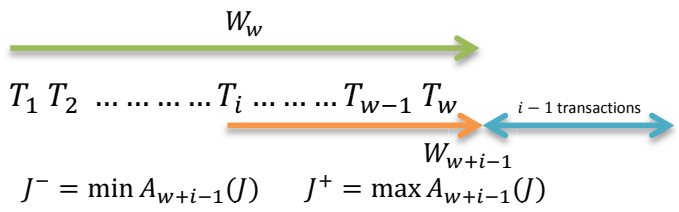

Given a window $W_{w}=T_{1} \cdots T_{w-1} T_{w}$, for every closed itemset $J$ in the candidate list $C_{i}(1 \leq i \leq w)$, the lower-bound and the upper-bound of the area of this itemset in any future sliding window containing $T_{i}$ are denoted $J^{-}$and $J^{+}$. The lower-bound $J^{-}$at time point $w$ is calculated as the area of $J$ in the transactions $T_{i}, T_{i+1}, \cdots, T_{w}$ and the upper-bound $J^{+}$is calculated as

$$
J^{+}=J^{-}+(i-1) \times|J|
$$

Proposition 1. $J^{-}$and $J^{+}$are the correct lower-bound and upper-bound on the area of $J$ in any future window $W_{t}$ of size $w, w \leq t$, containing the transaction $T_{i}$.

Example 2 (Bounds). Figure 2 (upper-part) shows an example of a sliding window with size $w=5$. Each candidate $J$ in a candidate list $C_{i}$ is associated with two numbers corresponding to the lower-bound and the upper-bound on the area of the candidate. For instance, for the candidate $J=a c$ associated with the 


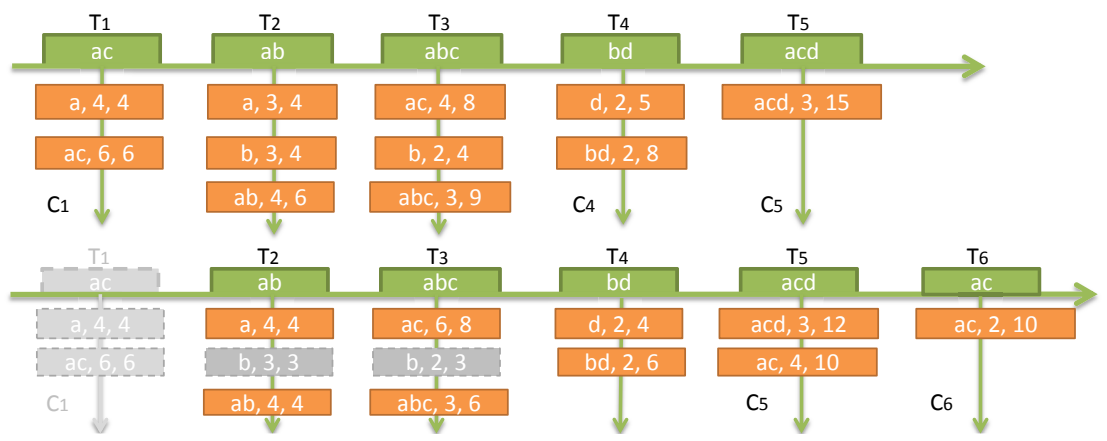

Fig. 2. An example of the summary maintained by the cTile algorithm for sliding windows with size $w=5$. The summary contains candidate itemsets which can become a top- $k$ tile in the future. Every candidate is associated with a lower-bound and an upper-bound on the area which are used to prune the candidate list.

candidate list $C_{3}$ the lower-bound is $J^{-}=4$ because the area of $a c$ in the set of currently observed transactions $T_{3}, \cdots, T_{5}$ is $2 * 2=4$. For any future window in which the transaction $T_{3}$ is not expired, the area of $a c$ is at least as large as 4 . Meanwhile $J^{+}=8$ because the area of $a c$ in any future window that contains $T_{3}$ is at most $4+2 * 2=8$. The area is equal to $J^{+}$only when the new transactions $T_{6}$ and $T_{7}$ both contain $a c$.

Algorithm 1 incrementally maintains the candidate lists $C_{i}$ in the summary. When a new transaction is appended to the end of the window, the bounds $J^{+}$and $J^{-}$are updated accordingly (line 10-13). Then the new transaction is intersected with every element in the summary and the intersections are added to the corresponding candidate list (line 8-9). If there exists another candidate $B \in C_{j}$ kept at a younger transaction $T_{j}(j>i)$, such that $B^{-}$is ranked $k^{t h}$ in all transactions younger than $T_{i}$ (including itself) and meanwhile $B^{-}>J^{+}$, then $J$ will never be among top- $k$ tiles in any future sliding window, hence $J$ is removed from the summary. When a transaction is expired, it is removed from the window along with the candidate list stored at the given transaction.

Example 3 (cTile). Figure 2 (bottom part) shows one update step of Algorithm 1 when a new transaction $T_{6}=\{a, c\}$ is appended to the window and $T_{1}$ together with $C_{1}$ are removed from the summary. First, $T_{6}$ is intersected with all the existing candidates in $C_{2}, C_{3}, \cdots, C_{5}$ to add new closed sets to the candidate lists. After that all the lower-bounds and the upper-bounds are updated accordingly.

Assume that we want to get top-3 largest tiles in the sliding window, i.e. $k=3$. Since $(b, 3,3)$ in $C_{2}$ has the upper-bound equal to 3 . It is removed from the summary because the itemset ranked at the third position in the candidates lists of younger transactions (including $\left.T_{2}\right)$ are $(a, 4,4),(a c, 4,10),(a b, 4,4)$ whose lower-bound is 4 . The same pruning operation can be applied for $(b, 2,3)$ in $C_{3}$.

Theorem 1. Given $k, w$ and a stream of transactions, using the summary in Algorithm 1 we can answer the top-k largest tiles exactly. 


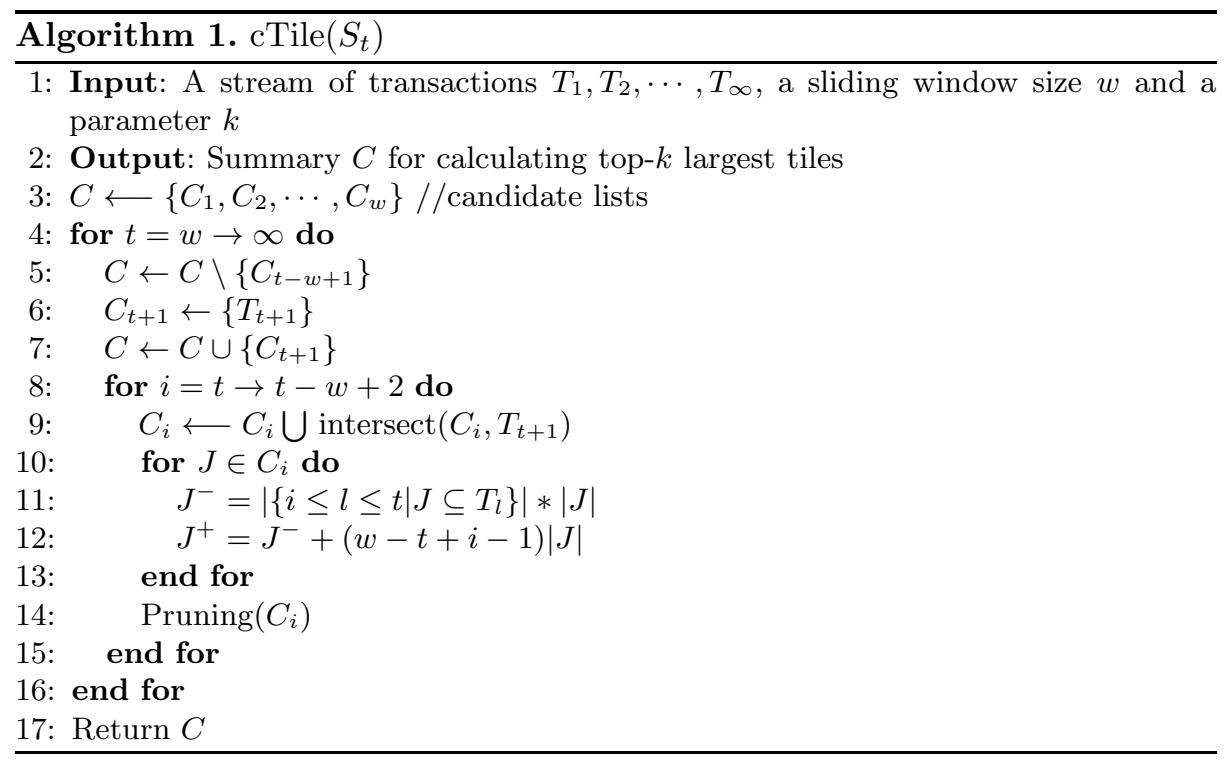

Proof. Assume that there exists a top- $k$ largest tile $I$ in a window $W_{t}$ not recognized as a top- $k$ largest tile (false negative). The first transaction of $W_{t}$ containing $I$ is denoted by $T_{i}$. False negative can only occur if at time $t, I \notin C_{i}$. Therefore either $I$ is directly pruned from $C_{i}$ by pruning criteria or indirectly pruned because its closed supersets are pruned.

Since the bounds are exact the former case cannot happen. The latter case happens when $I$ is not a closed itemset at the moment its closed supersets are pruned. In such case, the upper-bound of $I$ is always less than the upper-bound of its closed supersets which were used to prune the supersets. Therefore, $I$ is not a top- $k$ tile in $W_{t}$. Both cases lead to contradiction.

\subsection{Approximation Algorithm}

The size of the summary maintained by cTile grows quickly when the window size increases making it inefficient for monitoring large windows. The main reason is that each time a new transaction arrives, it has to be intersected with a large amount of candidates, which is a time-consuming operation. Therefore, in this section we discuss an approximation algorithm named aTile that approximates the set of largest tiles efficiently.

The main process of the approximation algorithm is almost the same as Algorithm 1. The only difference of these two algorithms lies in the method of candidate pruning. Instead of using an upper-bound on the area, aTile tries to approximate the future area of a tile. This estimate is used to prune the candidates in the same way as cTile does.

Let us denote the probability of observing an itemset $J$ as a subset of a transaction in the data stream by $\mu_{J}$. Therefore, the expectation of the area of the itemset $J$ in a window of size $w$ is $\mu_{J} \cdot|J| . w$. 
Given a window $W_{w}=T_{1} \cdots T_{w-1} T_{w}$, assume that $J$ is kept in the candidate list $C_{i}$ of transaction $T_{i}$. The lower-bound $J^{-}$is calculated as in the cTile algorithm. Instead of using an upper bound $J^{+}$on the area of $J$, we compute an estimate of the area of $J$ denoted by $J^{*}$.

Since $\mu_{J}$ is unknown, we cannot directly define $J^{*}$ as $\mu_{J} \cdot|J| . w$. The probability $\mu_{J}$ is thus estimated in the sub-window $T_{i} \cdots T_{w}$. However, if this sub-window is too small, the estimation of $\mu_{J}$ is not accurate. Therefore, we introduced a threshold $L \leq w$ : if the sub-window is smaller than $L$, we do not use this estimation and fall back on using the upper bound $J^{+}$for $J^{*}$ :

$$
J^{*}= \begin{cases}J^{-}+(i-1) \times|J| & \text { if } w-i+1 \leq L\left(\text { i.e., } J^{*}=J^{+}\right) \\ \frac{J^{-}}{(w-i+1)} \times w & \text { if } w-i+1>L\end{cases}
$$

All the steps of the of the aTile algorithm are very similar to the cTile algorithm except that it uses $J^{*}$ instead of $J^{+}$for candidate pruning. In the experiment section we empirically show that the aTile algorithm is more efficient than the cTile algorithm because its candidate set is more concise. An important property of the aTile algorithm is that the error rate on the accuracy of the result is bounded if we assume that the transactions are i.i.d. in a window of size $2 w$.

\section{Theoretical Analysis}

In this section, we show theoretical bounds on the probability of errors induced by the aTile algorithm. We mainly show that the error rate is extremely low when $L$ is large enough. We consider two types of error event: False negative $(F N)$ : in a window $W_{t^{*}}$ for some $t^{*}$, there is a true top- $k$ largest tile that is not present in the results returned by the aTile algorithm and False positive (FP): in a window $W_{t^{*}}$ for some $t^{*}$, there is a non top- $k$ largest tile that is present in the result returned by the aTile algorithm.

\subsection{False Negative Bound}

Let us assume that $I$ is a true top- $k$ largest tile in the window $W_{t^{*}}$ and $I_{k}^{t^{*}}$ is ranked at position $k$ in the list of a true top- $k$ largest tile of the window $W_{t^{*}}$. Let $\triangle \geq 0$ be the difference between the average area of $I$ and $I_{k}^{t^{*}}$ in the window $W_{t^{*}}$, i.e. $\triangle=\frac{A_{t^{*}}(I)-A_{t^{*}}\left(I_{k}^{t^{*}}\right)}{w}$. The following lemma show the relationship between the probability of a false negative, $L$ and $\triangle$ :

Lemma 1 If the transactions are independent and identically distributed (i.i.d) in a window of size $2 w$, the probability that a random top- $\mathrm{k}$ tile is not reported, is bounded as follows:

$$
\operatorname{Pr}(F N)<4 w * e^{-\frac{L \triangle^{2}}{2|I|^{2}}}
$$


Proof. A false negative happens in the window $W_{t^{*}}$ with respect to an itemset $I$ if its area is underestimated in that window. This only happens if there exists at least one moment $t<t^{*}$ such that $I$ is pruned from the candidate list $C_{i}$ $(t-w<i \leq t)$.

Given a time $t$, let $W_{0}$ be a window containing transactions $T_{i}, T_{i+1}, \cdots, T_{t}$ where $\left|W_{0}\right|=w_{o}>L$ and let $f_{0}(I)$ be the frequency of $I$ in the window $W_{0}$. When $t$ is given, the event " $I$ is removed from $C_{i}$ " (denoted by $R_{t}$ ) only happens when the estimate of the upper-bound on the area of $I$ is less than the area of $I_{k}^{t^{*}}$, i.e. $f_{W_{0}}(I)|I| \frac{w}{w_{0}}<A_{t^{*}}\left(I_{k}^{t^{*}}\right)$. Therefore, we have:

$$
\begin{aligned}
\operatorname{Pr}\left(R_{t}\right) & <\operatorname{Pr}\left(f_{0}(I)|I| \frac{w}{w_{0}} \leq A_{t^{*}}\left(I_{k}^{t^{*}}\right)\right)<\operatorname{Pr}\left(f_{0}(I)|I| \frac{w}{w_{0}}<A_{t^{*}}(I)-w \triangle\right) \\
& <\operatorname{Pr}\left(\frac{f_{0}(I)}{w_{0}}<\frac{f_{t^{*}}(I)}{w}-\frac{\triangle}{|I|}\right)<\operatorname{Pr}\left(\left|\frac{f_{0}(I)}{w_{0}}-\frac{f_{t^{*}}(I)}{w}\right|>\frac{\triangle}{|I|}\right) \\
& <\operatorname{Pr}\left(\left|\frac{f_{0}(I)}{w_{0}}-\mu_{I}\right|+\left|\frac{f_{t^{*}}(I)}{w}-\mu_{I}\right|>\frac{\triangle}{|I|}\right) \\
& <\operatorname{Pr}\left(\left|\frac{f_{0}(I)}{w_{0}}-\mu_{I}\right|>\frac{\triangle_{0}}{w_{0}}\right)+\operatorname{Pr}\left(\left|\frac{f_{t^{*}}(I)}{w}-\mu_{I}\right|>\frac{\triangle_{1}}{w}\right) \\
& <\operatorname{Pr}\left(\left|f_{0}(I)-\mu_{I} w_{0}\right|>\triangle_{0}\right)+\operatorname{Pr}\left(\left|f_{t^{*}}(I)-\mu_{I} w\right|>\triangle_{1}\right)
\end{aligned}
$$

Where $\triangle_{0}=\frac{\triangle w_{0}}{2|I|}$ and $\triangle_{1}=\frac{w \triangle}{2|I|}$. It is important to notice that $w_{0} \mu_{I}$ and $w \mu_{I}$ are the expectation of the frequency of $I$ in the window $W_{0}$ and the window $W_{t^{*}}$ respectively. Since the transactions are independent to each other, according to the Hoeffding inequality [1] we have:

$$
\operatorname{Pr}\left(\left|f_{0}(I)-w_{0} \mu_{I}\right|>\triangle_{0}\right)<2 e^{-\frac{2 \triangle_{0}^{2}}{w_{0}}}<2 e^{-w_{0} \frac{\triangle^{2}}{2|I|^{2}}}<2 e^{-L \frac{\triangle^{2}}{2|I|^{2}}}
$$

A similar inequality can be obtained for bounding the second term as follows:

$$
\operatorname{Pr}\left(\left|f_{t^{*}}(I)-w \mu_{I}\right|>\triangle_{1}\right)<2 e^{-\frac{2 \triangle_{1}^{2}}{w}}<2 e^{-w \frac{\Delta^{2}}{2|I|^{2}}}<2 e^{-L \frac{\Delta^{2}}{2|I|^{2}}}
$$

Moreover, since $F N$ happens only when there at least one moment $t$ such that $R_{t}$ happens, therefore:

$$
\operatorname{Pr}(F N)<\operatorname{Pr}\left(\cup_{t^{*}-w<t \leq t^{*}} R_{t}\right)<\sum_{t^{*}-w<t \leq t^{*}} \operatorname{Pr}\left(R_{t}\right)
$$

Inequalities [3, 4] and [5] prove the lemma.

A direct corollary of Lemma 1 is shown in the following theorem:

Theorem 2. If I is strictly more important than $I_{k}^{t^{*}}$, i.e. the expectation of the area of $I$ in a transaction is strictly greater than the expectation of the area of $I_{k}^{t^{*}}$ in a transaction and $L=O(w)$ then: $\lim _{L \longrightarrow \infty} \operatorname{Pr}(F N)=0$ 
Proof. Let $E(A(I))$ be the expectation of the area of $I$ in a transaction and $E\left(A\left(I_{k}^{t^{*}}\right)\right)$ be the expectation of the area of $I_{k}^{t^{*}}$ in a transaction. Since $\triangle=$ $\frac{A_{t^{*}}(I)-A_{t^{*}}\left(I_{k}^{t^{*}}\right)}{w}$ we can imply that:

$$
\frac{\triangle}{|I|}=\frac{A_{t^{*}}(I)-A_{t^{*}}\left(I_{k}^{t^{*}}\right)}{w|I|} \simeq \frac{E(A(I))-E\left(A\left(I_{k}^{t^{*}}\right)\right)}{|I|}
$$

The last equation is the result of the law of large number when $L$ goes to $\infty$. From the last equation we can imply that $\lim _{L \longrightarrow \infty} 4 w e^{-L \frac{\Delta^{2}}{2|I|^{2}}}=0$ from which the theorem is proved.

\subsection{False Positive Bound}

In this subsection we prove bound for false positive error which can be obtained in a similar way as the bound of false negative.

Let $J$ be an itemset that is not a true top- $k$ largest tile in the window $W_{t^{*}}$ but returned by the aTile algorithm as a false positive tile. An itemset ranked at position $k$ in the list of a true top- $k$ largest tile of the window $W_{t^{*}}$ is denoted by $I_{k}^{t^{*}}$. Let $\triangle \geq 0$ be the difference between the area of $J$ and $I_{k}^{t^{*}}$ in the window $W_{t^{*}}$, i.e. $\triangle=\frac{A_{t^{*}}\left(I_{k}^{t^{*}}\right)-A_{t^{*}}(J)}{w}$.

The following lemma show the relationship between the probability of a false positive and $L, \triangle$ (proof is similar to Lemma 11):

Lemma 2 If the transactions are i.i.d. in a window of size $2 w$ the probability of false positive, i.e. the event that a random non top- $\mathrm{k}$ tile is reported, is bounded as follows:

$$
\operatorname{Pr}(F P)<4 w * e^{-\frac{L \triangle^{2}}{2|I|^{2}}}
$$

A corollary of Lemma 2 is shown as follows (proof is similar to Theorem 2):

Theorem 3. If tile size is bounded and if $J$ is strictly less important than $I_{k}^{t^{*}}$, i.e. the expectation of the area of $J$ is strictly less than the expectation of the area of $I_{k}^{t^{*}}$ in a transaction and $L=O(w)$ then: $\lim _{L \longrightarrow \infty} \operatorname{Pr}(F P)=0$

Theorem 3 and Theorem 2 show an interesting result that the probability of false positive and false negative decrease exponentially with $L=O(w)$. It is important to notice that the condition $L=O(w)$ can be replaced by a weaker assumption $L=k \log w$ for some constant values $k$. If $k$ is large enough the bound is also closed to zero. Although the bounds are not tight, in experiments, we empirically show that false negative rate and false positive rate are negligible even when $L$ is set to a small value. 


\subsection{Long Lasting Tiles}

The two previous Theorems give probabilistic results. We can also show a deterministic one: if an itemset stays in the top- $k$ largest tiles for more than $w$ consecutive windows and its area is at least twice the area of the $k$-th tile, then the aTile algorithm finds it. An important point of this theorem is that it does not depends on the value of $L$. Therefore, even if the probability of false negative or false positive is higher with a small $L$, the algorithm is still able to mine these tiles that we call the long lasting tiles.

Theorem 4. Let $z$ be a time and $J$ be an itemset. If the area of $J$ in every windows $W_{t}$ with $z \leq t<z+w$ is larger than two time the area of the $k$-th largest tile in this window (i.e., $A_{t}(J) \geq 2 A_{t}\left(I_{k}^{t}\right)$ ), then there is a time $z-w<t^{*}<z+w$ such that $J$ (or one of its supersets) is in $C_{t^{*}}$ and is never pruned by aTile.

Proof. We define hypothesis $\mathrm{H}$ as: $t^{*}$ does not exist. We will show that if $\mathrm{H}$ is true, it leads to a contradiction and thus the theorem must be true. We denote by $[x, y]$ the subsequence of transactions $T_{x} \cdots T_{y}$. The occurrence times of $J$ in the subsequence $[z-w+1, z+w-1]$ are denoted by $z-w<t_{1}<\ldots<t_{k}<z+w$.

If $\mathrm{H}$ is true, then for every $t_{i}$, itemset $J$ and its supersets must be pruned from $C_{t_{i}}$ at some time $t_{i}^{\prime}$ no later than $t_{i}+w-1$, i.e., $t_{i}^{\prime}<t_{i}+w$.

Let $S_{i}$ be the subsequence $\left[t_{i}, t_{i}^{\prime}\right]=T_{t_{i}} \cdots T_{t_{i}^{\prime}}$. If for all $z-w<t_{i} \leq z$, we have $t_{i}^{\prime} \leq z$ then we define $W_{0}=W_{z}$. Otherwise, we take $W_{0}=W_{t_{\text {max }}^{\prime}}$ where $t_{\text {max }}^{\prime}=\max \left\{t_{i}^{\prime} \mid t_{i} \leq z\right\}$.

We now construct a set $\mathcal{S}$ of these subsequences $S_{i}$ such that every subsequence of $\mathcal{S}$ is included in $W_{0}$, every occurrence of $J$ is in at least one of these subsequences, and at most two of these subsequences intersect at any given time. We start with $\mathcal{S}=\emptyset$ if $W_{0}=W_{z}$ or with $\mathcal{S}=\left\{\left[t_{\max }, t_{\text {max }}^{\prime}\right]\right\}$ otherwise. We scan the window $W_{0}$ from left to right. If there is an occurrence of $J$ at time $t_{i}$ not already in a subsequence of $S$, then we add $S_{i}$ in $\mathcal{S}$. All the added subsequences are disjoint by construction, only the last added one may intersect with $\left(t_{\max }, t_{\max }^{\prime}\right)$.

For every $S_{i} \in \mathcal{S}$, let $A_{i}$ be the area of the $k$-th largest tile of $S_{i}$ used to prune $J$, i.e., $J^{*}<A_{i}$. Since by Eq. $2 J^{*} \geq w A_{S_{i}}(J) /\left(t_{i}^{\prime}-t_{i}+1\right)$, we have $w A_{S_{i}}(J) /\left(t_{i}^{\prime}-t_{i}+1\right)<A_{i}$. If we define $A_{m}=\max A_{i}$, then $A_{S_{i}}(J)<A_{m}\left(t_{i}^{\prime}-\right.$ $\left.t_{i}+1\right) / w$ and by summation for all $S_{i} \in \mathcal{S}: \sum_{i} A_{S_{i}}(J)<A_{m} \sum_{i}\left(t_{i}^{\prime}-t_{i}+1\right) / w$. Since all occurrences of $J$ are covered by at least one $S_{i}, \sum_{i} A_{S_{i}}(J)>A_{W_{0}}(J)$ and since at most two subsequences $S_{i}$ from $\mathcal{S}$ intersect at any given time, $\sum_{i}\left(t_{i}^{\prime}-t_{i}+1\right) \leq 2 w$ and thus $A_{W_{0}}(J)<2 A_{m}$. Since $A_{m}$ is the size of the $k$-th tile of one of the $S_{i} \subseteq W_{0}$, it is less than the size of the $k$-th tile in the whole window $W_{0}$. Finally, $A_{W_{0}}(J)$ is strictly less than two time the area of the $k$-th tile in $W_{0}$ which is a contradiction.

\section{Experiments}

In this section, we perform experiments with two real-life data streams to compare the proposed algorithms to the baseline approaches with respect to the efficiency and the accuracy of the results. The two datasets are: 

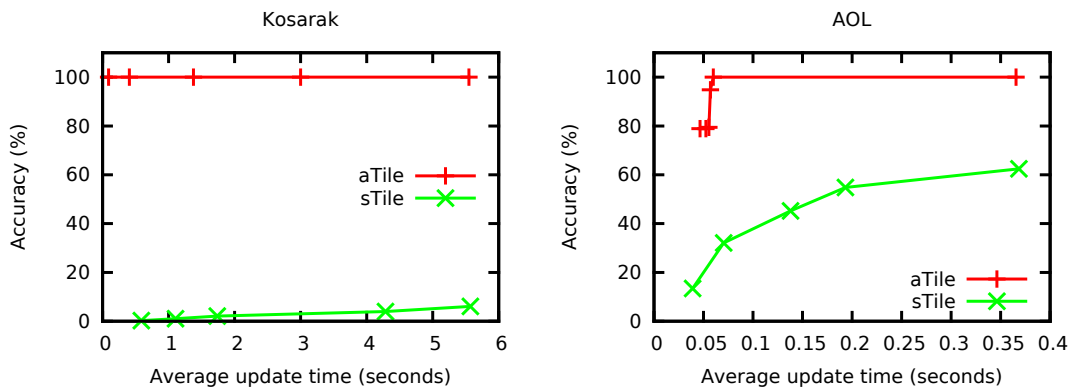

Fig. 3. Accuracy versus average update time in seconds of the aTile algorithm and the sTile algorithm when $\mathrm{L}$ and the number samples increases
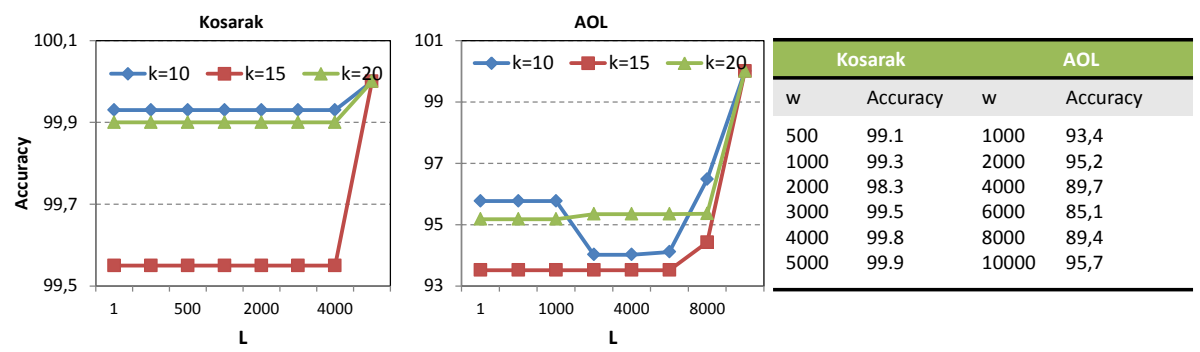

Fig. 4. The average accuracy of the aTile algorithm when $L$ is varied. In the table: the average accuracy of the top-10 largest tiles returned by aTile when the window size is varied and $L=0.1 w$

- Kosarak: 1M transactions of click log by the users of a website. Item are a pages in the website. The largest transaction contains 500 items.

- AOL: 100K search queries by the users of the AOL search engine. Each item is a keyword in the search query. Most queries are short and the longest query contains only 26 keywords.

The datasets and the source codes of aTile and cTile in $\mathrm{C}++$ are available for download 2 . The baseline algorithms for comparison are as follows:

- Tile: the original implementation of the tiling algorithm for static database [7. In order to adopt this algorithm for a data stream, we use Tile to recalculate the top- $k$ largest tiles from scratch whenever the window is sliding.

- sTile: a sampling based technique proposed in 2. The sTile algorithm samples $N$ itemsets from the window such that each itemset is sampled with probability proportional to the area of the itemset. The top- $k$ largest tiles are extracted from the samples every time the window is sliding.

2 http://www.win.tue.nl/ lamthuy/tile.htm 


\begin{tabular}{llll}
\hline \multicolumn{2}{c}{ sTile } & \multicolumn{2}{c}{ aTile } \\
\hline \# samples & Sum of area & L & Sum of area \\
5000 & 2652 & 1000 & 20384 \\
10000 & 2753 & 2000 & 20384 \\
20000 & 2822 & 3000 & 20384 \\
40000 & 2918 & 4000 & 20384 \\
80000 & 2992 & 5000 & 20388
\end{tabular}

Fig. 5. The average sum (larger is better) of the area of the top-10 tiles returned by the aTile and the sTile algorithms
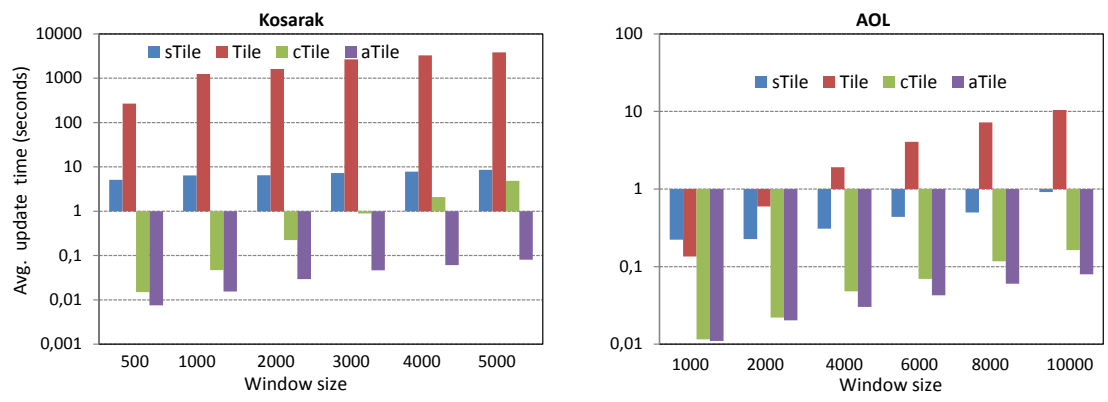

Fig. 6. Average time per update according to the window size

\subsection{Accuracy}

We performed experiments to demonstrate the effectiveness of the aTile algorithm in term of accuracy. In the first experiment the window size is set to $w=5000$ for the Kosarak dataset and $w=10000$ for the AOL dataset. Figure 4 shows the average accuracy calculated as the precision of the top- $k$ largest tiles returned by the aTile algorithm when $L$ is varied. Even for very small $L$, the accuracy is very high, e.g. $99 \%$ in the Kosarak dataset and $93 \%$ in the AOL dataset. The accuracy increases and reaches $100 \%$ accuracy when $\mathrm{L}$ is increased. In the same figure, we can also see that the results are similar when $k$ is varied.

In order to compare to the baseline algorithm sTile, we plot the accuracy (y-axis) versus average update time (x-axis) in Figure 3. We varied both $L$ for the aTile algorithm and the number of samples of the sTile algorithm. Recall that the sTile algorithm depends on the number of samples it collects from the window. When the number of samples increases, so do the accuracy and the update time. This fact is illustrated in Figure 3 in which the sTile algorithm is significantly less accurate than the aTile algorithm given about the same average update time.

The accuracy of sTile may be negatively influenced by the fact that sTile has a very low probability to find each top- $k$ tile. When we calculated the average sum of the area of the top-10 tiles in the Kosarak dataset for $w=5000$, we observed that it was significantly lower for sTile than for the aTile algorithm (Figure 5). This confirmed that sTile is not able to find the large tiles with a reasonable number of samples. 

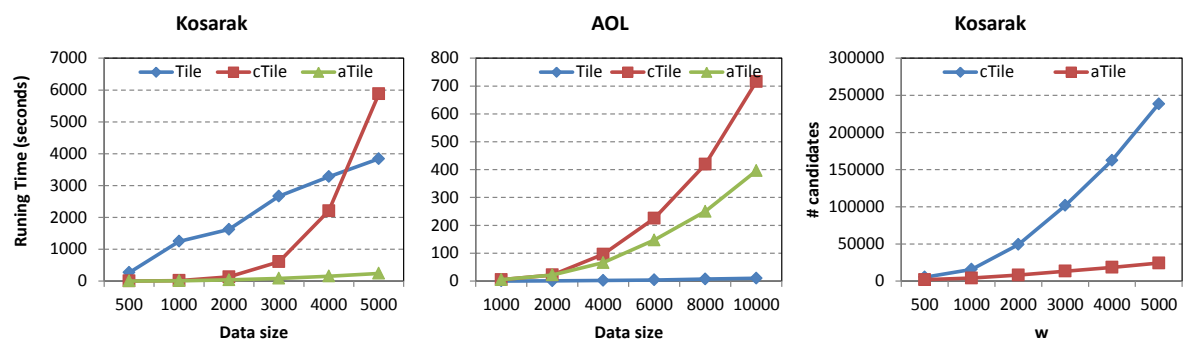

Fig. 7. Running time according to the database size when the algorithm is used to mine large tiles in a database instead of a stream. The right most subplot shows the number of candidates in the summary when the window size increases.

\subsection{Efficiency}

Figure [ shows average update time of four algorithms for $k=10$ and different values of $w$. The number of samples in the sTile algorithm was set to $N=80000$ (when $N$ is larger, the algorithm become significantly slower). We set $L=\frac{w}{10}$ (as shown in Sect. 6.1, the accuracy is then always above 85\%). In term of update time, aTile is up to an order of magnitude (50x) faster than the cTile algorithm and about two to three orders of magnitude faster than the sTile (100x) and the Tile $(1000 \mathrm{x})$ algorithms. The speed-up increases with the window size.

In Figure 7, the last plot shows the number of candidates kept in the summary of the aTile and the cTile algorithm for varying values of $w$ (the result of the AOL dataset is omitted because it is very similar to the results of the Kosarak dataset in this experiment). The aTile algorithm is more memory efficient than the cTile algorithm as the number of candidates it keeps in the summary is much lower.

Finally, in Figure 7 we show the running time when the aTile, cTile and Tile algorithms are used to find the top- 10 largest tiles in a static corpus with varying size. The purpose of the experiment is to see whether the aTile and the cTile algorithm can find the large tiles in a static corpus more efficiently than the Tile algorithm. For the AOL dataset, when most of transactions are small, the Tile algorithm is the fastest one. However, for the Kosarak dataset, when the average transaction size is larger, aTile outperforms both Tile and cTile. Therefore, the aTile algorithm can not only be used for mining large tiles from a data stream but also to mine large tiles from a database efficiently.

\subsection{Application to Topics Monitoring in the AOL Query Stream}

In order to show a potential application of the work we created a demo vided 3 to visualize the top- $k$ largest tiles of the AOL query stream. Each snapshot of the video corresponds to a list of the largest tiles extracted from a sliding window.

3 http://www $\cdot$ youtube.com/watch?v=3UCjs9d91_g 

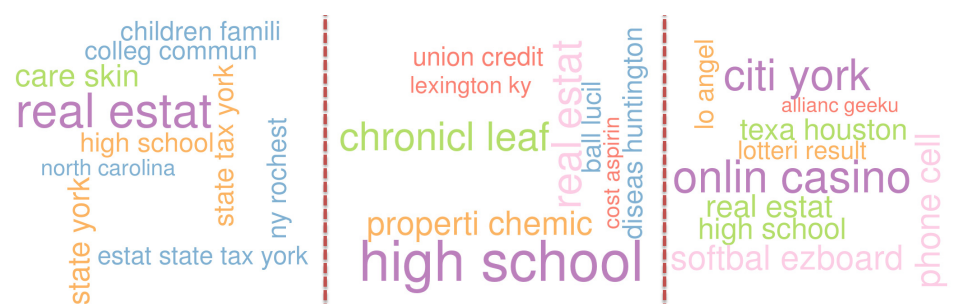

Fig. 8. Top-10 largest tiles in windows (ordered by timestamps) from the AOL query stream. Larger words correspond to tiles with bigger area. The tile "high school" emerges in the second window related to searches for the "high school musical movies".

Figure 8 shows example snapshots taken from the demo video. Three snapshots of the demo (ordered by timestamps) show the evolution of the largest tiles overtime. Each snapshot is visualized by the wordcloud tool in $R$. Larger words correspond to tiles with larger area.

For example, in the first snapshot the keyword "real estate" is the most important tile. However, it becomes less important in the second and the third snapshot. Meanwhile, new important tiles such as "high school" is emerging as search for the "high school musical movies" increases in 2006. With this demo, users can track the dynamic of important search topics online.

\subsection{Application to Tracking in Videos}

We investigate how mining top- $k$ largest tiles in a data stream can be useful for analysis of videos and, in particular, for tracking. A more detailed description of this application can be found in [3. We worked on a real video made of 5619 frames. This video is shot from a car while following another car (the main object). The main object is present in almost all the frames of the video. If we are able to represent the video as a data stream and use our tile mining algorithm on it, it should discover the car as a large tile or a set of large tiles.

We used the segmentation algorithm 4 , 8] to generate a stream of graphs (one graph per frame). Each graph is a Region Adjacency Graph (RAG) where each node is a region (a set of adjacent pixels) and two nodes are connected if their regions are adjacent. This algorithm performs a temporal segmentation, which means that a given region (and thus, the corresponding node) is present in several successive frames. However, a single region is not enough to track an object in the video: due to change in the object pose or illumination, some regions will split, or merge or disappear and so do the corresponding nodes. We then build a transaction consisting in the set of nodes of each RAG.

Mining tiles on this data stream results in tiles containing regions spread all over the frames. Moreover, the top-k tiles are generally very similar (only differing by one or two regions). Indeed, by removing the information about the

4 http://www.videosegmentation.com 


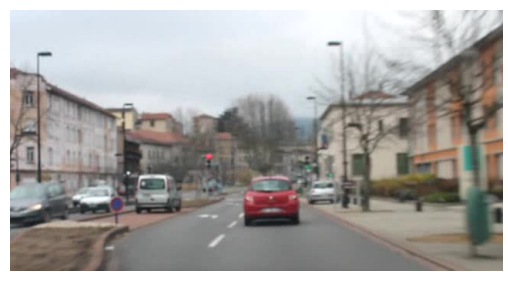

Fig. 9. Frame of the video

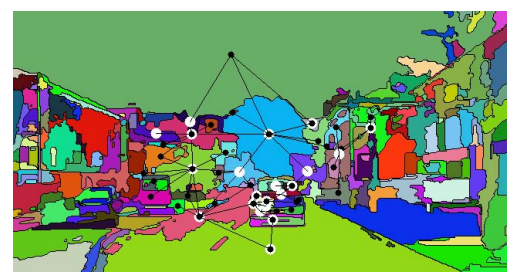

Fig. 10. top-30 star-shaped tiles in the segmented frame

adjacency of the regions, it was not possible to find meaningful tiles. The main problem is that the regions composing the car (or more generally, an object to be tracked) must be "close" to each other and this was not taken into account in the original setting. Therefore, we added a spatial constraint to the problem. We chose to mine tiles that are star subgraphs of the RAGs. A star subgraph is a graph with nodes $i_{0}, i_{1}, \ldots i_{n}$ where $i_{1}, \ldots, i_{n}$ are nodes adjacent to $i_{0}$ in the RAG. This formalization ensures that the items in a tile are adjacent to a "center" item $i_{0}$. Moreover, it is easy to integrate this constraint into our algorithms: the intersection of two star graphs with the same center is just the intersection of their set of nodes. The algorithm is otherwise unchanged.

We extracted the top-30 tiles with a window of 300 frames (see an example on Fig 10). Then we checked if the extracted tiles could be useful to track the main object (car). We manually drawn the bounding box containing the car in each fifth frame. Then, we selected, for each frame, the tile with the best precision. We end up with a set of 69 tiles with an overall precision of 0.92 and recall of 0.74 on the whole video (and even a recall of 1 on the 2000 first frames). This means that the tiles can actually be used as a high level feature for tracking. Of course, in a real application, the bounding box is not known. But techniques like those described in [5] can be used to build tracks from the extracted tiles.

\section{Conclusions and Future Works}

In this paper, we proposed two algorithms for mining the top- $k$ largest tiles from a data stream with a sliding window model. The first candidate-based algorithm cTile solves the problem exactly but the update time becomes important when the window size increases. The second one is an approximation algorithm with theoretical bounds on the error rate. Experiments with two real-life datasets show that the approximation algorithm can find large tiles with high accuracy while being an order of magnitude faster than the candidate based algorithm and two to three order of magnitude faster than the other baseline algorithms.

We also show potential applications of large tiles mining in monitoring emerging popular topics in a search engine query log or in a tracking problem. A possible extension for future work is to consider mining different types of tiles with constraints for meaningful applications. 
Acknowledgements. This work is supported by the Netherlands Organization for Scientific Research (NWO) through the project Mining Complex Patterns in Stream (COMPASS) and the French ANR project SoLStiCe (ANR-13-BS020002-01). We would like to thank Dr. Bart Goethals for providing us the implementation of the Tile algorithm and Pr. Toon Calders for his precious comments.

\section{References}

1. Aggarwal, C.C. (ed.): Data Streams - Models and Algorithms. Advances in Database Systems, vol. 31. Springer (2007)

2. Boley, M., Lucchese, C., Paurat, D., Gärtner, T.: Direct local pattern sampling by efficient two-step random procedures. In: KDD, pp. 582-590 (2011)

3. Calders, T., Fromont, É., Jeudy, B., Lam, H.T.: Analysis of videos using tile mining. In: Real-World Challenges for Data Stream Mining Workshop (2013)

4. Cerf, L., Besson, J., Nguyen, K.N., Boulicaut, J.F.: Closed and noise-tolerant patterns in n-ary relations. Data Min. Knowl. Discov. 26(3), 574-619 (2013)

5. Diot, F., Fromont, E., Jeudy, B., Marilly, E., Martinot, O.: Graph mining for object tracking in videos. In: Flach, P.A., De Bie, T., Cristianini, N. (eds.) ECML PKDD 2012, Part I. LNCS, vol. 7523, pp. 394-409. Springer, Heidelberg (2012)

6. Fernando, B., Fromont, E., Tuytelaars, T.: Effective use of frequent itemset mining for image classification. In: Fitzgibbon, A., Lazebnik, S., Perona, P., Sato, Y., Schmid, C. (eds.) ECCV 2012, Part I. LNCS, vol. 7572, pp. 214-227. Springer, Heidelberg (2012)

7. Geerts, F., Goethals, B., Mielikäinen, T.: Tiling databases. In: Suzuki, E., Arikawa, S. (eds.) DS 2004. LNCS (LNAI), vol. 3245, pp. 278-289. Springer, Heidelberg (2004)

8. Grundmann, M., Kwatra, V., Han, M., Essa, I.: Efficient hierarchical graph-based video segmentation. In: CVPR (2010)

9. van Leeuwen, M., Knobbe, A.J.: Diverse subgroup set discovery. Data Min. Knowl. Discov. 25(2), 208-242 (2012)

10. Lu, H., Vaidya, J., Atluri, V.: Optimal boolean matrix decomposition: Application to role engineering. In: ICDE, pp. 297-306 (2008)

11. Motwani, R., Raghavan, P.: Randomized algorithms. Cambridge University Press, USA (1995)

12. Pasquier, N., Bastide, Y., Taouil, R., Lakhal, L.: Discovering frequent closed itemsets for association rules. In: Beeri, C., Bruneman, P. (eds.) ICDT 1999. LNCS, vol. 1540, pp. 398-416. Springer, Heidelberg (1998)

13. Remmerie, N., Vijlder, T.D., Valkenborg, D., Laukens, K., Smets, K., Vreeken, J., Mertens, I., Carpentier, S.C., Panis, B., Jaeger, G.D., Blust, R., Prinsen, E., Witters, E.: Unraveling tobacco by-2 protein complexes with $\{\mathrm{BN}\}$ page $/ \mathrm{lcms} / \mathrm{ms}$ and clustering methods. Journal of Proteomics 74(8), 1201-1217 (2011)

14. Smets, K., Vreeken, J.: The odd one out: Identifying and characterising anomalies. In: SDM, pp. 804-815 (2011)

15. Tatti, N., Vreeken, J.: Discovering descriptive tile trees by mining optimal geometric subtiles. In: Flach, P.A., De Bie, T., Cristianini, N. (eds.) ECML PKDD 2012, Part I. LNCS, vol. 7523, pp. 9-24. Springer, Heidelberg (2012)

16. Vaidya, J., Atluri, V., Guo, Q.: The role mining problem: A formal perspective. ACM Trans. Inf. Syst. Secur. 13(3) (2010)

17. Vreeken, J., van Leeuwen, M., Siebes, A.: Krimp: mining itemsets that compress. Data Min. Knowl. Discov. 23(1), 169-214 (2011) 